AGEING

\title{
Longer life through an odd Pol enzyme
}

The evolutionarily conserved enzyme RNA polymerase III is a driver of protein synthesis and cell growth. It emerges that partial suppression of this essential enzyme extends lifespan in yeast, roundworms and flies.

\section{BRUCE A. EDGAR \& SAVRAJ S. GREWAL}

$\mathrm{G}$ ene transcription in animal and fungal cells is accomplished by three RNA polymerase (Pol) enzymes. Although Pol II, which transcribes DNA to make messenger RNAs, is arguably the best understood, up to $80 \%$ of transcription is driven by Pol I and Pol III (ref. 1), known as the 'odd' Pols, which synthesize core RNA components of the cell's protein-synthesizing machinery. Pol I makes large ribosomal RNAs that form the structural and catalytic heart of the ribosome (the complex that mediates mRNA translation), whereas Pol III makes transfer RNAs also involved in translation, another ribosomal RNA, and small nuclear RNAs that mediate mRNA processing. Owing to its perceived unglamorous, albeit vital role, research on Pol III has been sparse - particularly with regard to organismal physiology. But in a paper online in Nature, Filer et al. ${ }^{2}$ highlight a role for this enzyme as a key determinant of cellular and organismal ageing.

Limiting an animal's metabolic rate by restricting its $\operatorname{diet}^{3}$ is one of the most effective and widespread ways to extend life. The benefits of dietary restriction have been traced to two nutrition-responsive signalling systems that regulate diverse aspects of cell metabolism - insulin signalling and mechanistic target-of-rapamycin (mTOR) signalling. One process that both of these pathways regulate is protein synthesis, and treatments that suppress (but don't block) protein synthesis can also extend lifespan in animals ${ }^{4,5}$.

Filer $e t$ al. extend this line of investigation to uncover a role for Pol III in lifespan. The authors first used the yeast Saccharomyces cerevisiae to demonstrate that the degradation of an essential subunit of Pol III, C160, extends a yeast cell's chronological lifespan (the lifespan of stationary, non-dividing cells). They then showed that partial depletion of the same Pol III subunit in the cells of nematode worms (Caenorhabditis elegans) or fruit flies (Drosophila melanogaster) similarly extends lifespan (Fig. 1).

Previous studies have shown that invertebrate lifespan can be extended by slowing metabolism specifically in the gut ${ }^{6}$. Filer et al. therefore inhibited Pol III in the guts of nematodes and the intestinal stem cells of flies. This sufficed to extend lifespan in both animals. In the fly, the authors showed that Pol III inhibition led to reduced protein synthesis, providing a probable mechanism for lifespan extension.

In Drosophila, death from gut dysfunction has been traced to a condition called agedependent dysplasia, in which the intestinal stem cells of aged flies over-proliferate and fail to differentiate properly in response to increasing oxidative stress from the bacterial contents of the gut ${ }^{6}$. This generates a defective intestinal lining, which eventually fails as a barrier to gut microbes, resulting in sepsis and death. Interestingly, age-dependent gut dysplasia can be delayed in flies by slowing the metabolism and division of intestinal stem cells - for example, by suppressing insulin or mTOR signalling, or through dietary restriction ${ }^{6}$. Filer et al. showed that Pol III depletion also delays age-dependent gut dysplasia.

Whether hyperactive intestinal stem cells contribute to human ageing is doubtful, but high rates of stem-cell proliferation pose other hazards. For instance, cancer risks are highly correlated with rates of stem-cell division in a cancer's tissue of origin ${ }^{7}$, and excessive cell division is typical in chronic inflammation ${ }^{8}$. Filer and colleagues' observations suggest that limiting Pol III activity in stem cells might reduce the risk of both of these common maladies.

Suppression of the evolutionarily conserved protein kinase enzyme mTOR, which is at the heart of the nutrient-responsive mTOR signalling pathway, is one of the most thoroughly tested ways to extend lifespan. Although mTOR regulates many aspects of cell metabolism, one of its central functions is to promote protein synthesis. Thus, it is logical to expect that lifespan extension by mTOR inhibition might stem from reduced protein synthesis. Most of the relevant studies have focused on mTOR substrates that modulate translation initiation. But, through a final set of experiments, Filer and colleagues provided

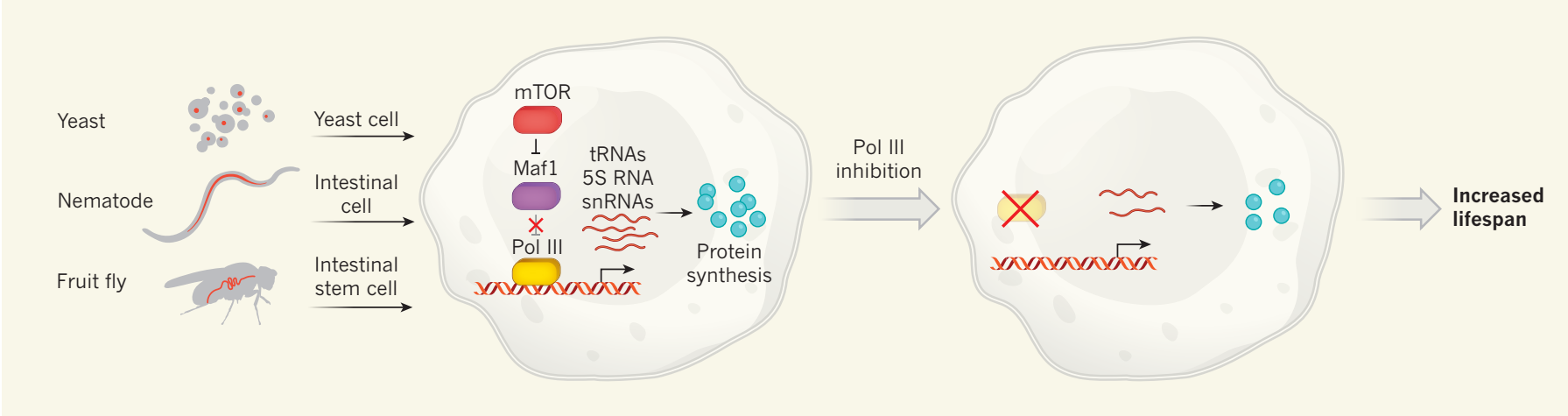

Figure 1 An evolutionarily conserved pathway modulating lifespan. Filer et al. ${ }^{2}$ have examined the role of the enzyme RNA polymerase III ( $\mathrm{Pol}$ III) in yeast, in the intestinal cells of nematode worms and in the intestinal stem cells of fruit flies. Under normal conditions, the metabolic enzyme mTOR promotes the activity of Pol III by inhibiting a repressor of Pol III, the protein Maf1. Active Pol III drives transcription of transfer RNAs (tRNAs), a ribosomal RNA (5S RNA) and small nuclear RNAs (snRNAs) - key parts of the cell's protein-synthesis machinery. The authors showed that inhibition of Pol III reduces protein synthesis and extends organismal lifespan. 
several lines of evidence to indicate that the effects of mTOR on lifespan might be attributed largely to its ability to regulate Pol III.

Here, the trail is fresh and exciting. It is known ${ }^{9}$ that mTOR regulates Pol III by inhibiting a Pol III transcriptional co-repressor protein, Maf1, and in the past five years, several studies have indicated that Mafl can alter cell and animal physiology in ways that might affect lifespan ${ }^{10-13}$. Most of these reports are consistent with Filer and colleagues' suggestion that mTOR affects ageing through Maf1, Pol III and protein synthesis, but Maf1 loss in worms had the opposite effect and actually extended lifespan ${ }^{12}$. This raises doubt as to whether ageing mechanisms are really so similar in different animals. But we should not expect them to be; the complex relationship between cellular metabolism, physiology and mortality clearly varies from species to species, and will need to be understood differently in each.

Looking beyond Pol III, one study ${ }^{14}$ has shown that both synthesis of ribosomal RNAs and the size of a nuclear structure called the nucleolus, in which ribosomes are assembled, diminish as flies age, and another ${ }^{15}$ reported a correlation between small nucleoli and longevity. Furthermore, suppression of ribosomal-RNA processing and nucleolar function can extend lifespan in worms and flies ${ }^{14,15}$. This suggests that various interventions that reduce ribosome production or function might help to stave off age-related pathologies. Given the complexity of the protein-synthesis machinery, there should be scores, if not hundreds, of molecules that might be targeted to suppress protein synthesis and extend lifespan. But a bonanza of promising molecular targets does not mean that translation to the pharmacy will be fast. As evidenced by various trials involving mTOR inhibitors, understanding which cells to suppress to combat a given condition, and how to hit them precisely and sufficiently without stopping them dead, remains a major challenge.

Bruce A. Edgar is in the Department of Oncological Sciences, Huntsman Cancer Institute, University of Utah, Salt Lake City, Utah 84112, USA. Savraj S. Grewal is in the Department of Biochemistry and Molecular
Biology, University of Calgary, Calgary, Alberta T2N 4N1, Canada. e-mails:bruce.edgar@hci.utah.edu; grewalss@ucalgary.ca

1. Warner, J. R. Trends Biochem. Sci. 24, 437-440 (1999).

2. Filer, D. et al. Nature http://dx.doi.org/10.1038/ nature25007 (2017).

3. McCay, C. M., Crowell, M. F. \& Maynard, L. A. J. Nutr. 10, 63-79 (1935)

4. Kapahi, P. Adv. Exp. Med. Biol. 694, 30-37 (2010),

5. Mehta, R., Chandler-Brown, D., Ramos, F. J., Shamieh, L. S. \& Kaeberlein, M. Adv. Exp. Med. Biol. 694, 14-29 (2010).

6. Wang, L., Karpac, J. \& Jasper, H. J. Exp. Biol. 217, 109-118 (2014).

7. Tomasetti, C. \& Vogelstein, B. Science $\mathbf{3 4 7}, \mathbf{7 8 - 8 1}$ (2015).

8. Li, H. \& Jasper, H. Dis. Model. Mech. 9, 487-499 (2016).

9. Michels, A. A. Biochem. Soc. Trans. 39, 487-491 (2011).

10.Rideout, E. J., Marshall, L. \& Grewal, S. S. Proc. Natl Acad. Sci. USA 109, 1139-1144 (2012).

11.Khanna, A., Johnson, D. L. \& Curran, S. P. Cell Rep. 9, 2180-2191 (2014).

12.Cai, Y. \& Wei, Y.-H. Oncotarget 7, 10812-10826 (2016).

13.Cai, Y. \& Wei, Y.-H. Aging 7, 133-143 (2015).

14.Demontis, F., Patel, V. K., Swindell, W. R. \& Perrimon, N. Cell Rep. 7, 1481-1494 (2014).

15.Tiku, V. et al. Nature Commun. 8, 16083 (2017) 